\title{
Use of Forward
} Sensitivity Analysis Method to Improve Code Scaling, Applicability, and Uncertainty (CSAU) Methodology

\section{NUTHOS-8}

\author{
Haihua Zhao \\ Vincent A. Mousseau
}

The INL is a

U.S. Department of Energy

National Laboratory

operated by

Battelle Energy Alliance

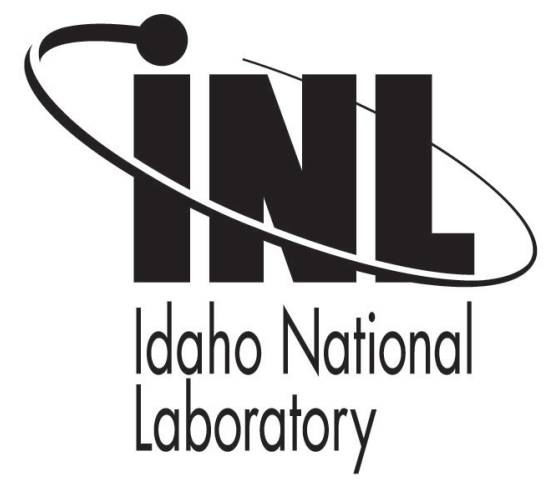

This is a preprint of a paper intended for publication in a journal or proceedings. Since changes may be made before publication, this preprint should not be cited or reproduced without permission of the author. This document was prepared as an account of work sponsored by an agency of the United States Government. Neither the United States Government nor any agency thereof, or any of their employees, makes any warranty, expressed or implied, or assumes any legal liability or responsibility for any third party's use, or the results of such use, of any information, apparatus, product or process disclosed in this report, or represents that its use by such third party would not infringe privately owned rights. The views expressed in this paper are not necessarily those of the United States Government or the sponsoring agency. 


\title{
Use of Forward Sensitivity Analysis Method to Improve Code Scaling, Applicability, and Uncertainty (CSAU) Methodology
}

\author{
Haihua Zhao and Vincent A. Mousseau \\ Idaho National Laboratory \\ PO BOX 1625, Idaho Falls, ID 83415-3870, USA \\ Haihua.zhao@inl.gov, Vincent.mousseau@inl.gov
}

\begin{abstract}
Since the Code Scaling, Applicability, and Uncertainty (CSAU) methodology was proposed about two decades ago, it has been widely used for new reactor designs and existing LWRs power uprates. In spite of these huge successes, CSAU has been criticized for the need of further improvement, focusing on two main issues - lack of objectiveness and high cost. With the effort to develop next generation safety analysis codes, new opportunities appear to take advantage of new numerical methods, better physical models, and modern uncertainty qualification methods. Forward sensitivity analysis (FSA) directly solves the partial differential equations for parameter sensitivities. Moreover, our work shows that time and space steps can be treated as special sensitivity parameters so that numerical errors can be directly compared with physical uncertainties. When the FSA is implemented in a new advanced system analysis code, CSAU could be significantly improved by quantifying numerical errors and allowing a quantitative PIRT (Q-PIRT) to reduce subjective judgement and improve efficiency. This paper will review the issues related to the current CSAU implementations, introduce FSA, show a simple example to perform FSA, and discuss potential improvements on CSAU with FSA. Finally, the general research direction and requirements to use FSA in an advanced system analysis code will be discussed.
\end{abstract}

\section{KEYWORDS}

CSAU, Forward sensitivity analysis, Q-PIRT

\section{OVERVIEW OF CSAU AND BEPU}

Code Scaling, Applicability, and Uncertainty (CSAU) methodology was developed in late 1980s by USNRC (Nuclear Regulatory Commission) to systematically quantify reactor simulation uncertainty. This method was developed in response to the USNRC rule change to allow the use of realistic physical models to analyze the loss of coolant accident (LOCA) in a light water reactor. [1] Prior to this time, the evaluation of this accident was subject to a prescriptive set of rules set by Appendix K of the regulations which require conservative models and assumptions to be applied simultaneously, leading to very pessimistic estimates of the impact of this accident on the reactor safety. The rule change therefore promised to provide significant benefits by allowing nuclear power reactor to increase output without major plant modifications. CSAU was developed to apply realistic methods, while properly taking into account uncertainty in data, physical modeling and plant variability.

The method was first demonstrated in 1996 for licensed application by Westinghouse to be structured, traceable, and practical [2]. Since then, Best Estimate Plus Uncertainty (BEPU) methods have been extensively used by the nuclear power industry around the world for power upratings, license renewals, and new design certifications. One example is AREVA's 
realistic large break LOCA (LB-LOCA) analysis methodology which received approval by USNRC in April 2003. [3] It incorporates the nonparametric statistical approach originally incorporated in the Gesellschaft fur Anlagen und Reaktorsicherheit (GRS) methodology for LOCA analysis. Another example is the Westinghouse automated statistical treatment of uncertainty method (ASTRUM) approved at the end of 2004. [4] The ASTRUM uses the same code and uncertainty distributions as the 1996 BELOCA method but uses nonparametric order statistics and more explicit treatment of more uncertainty parameters. For the same PWR plants, the calculated 95th percentile PCT (peak clad temperature) value is reduced by $126 \mathrm{~K}$ with the ASTRUM method, comparing with the value obtained from the 1996 BELOCA method. Almost all of demonstration applications of BEPU methods so far are for LOCA including both LB-LOCA and SB-LOCA (small break LOCA) and most of licensing applications of BEPU methods are for LBLOCA [5].

\subsection{Major Steps of CSAU}

In developing CSAU, the emphasis was placed on providing a practical engineering approach that could be used to quantify code uncertainties. [1] Consequently, for a specified plant and a given scenario, the CSAU method focuses only on important processes and/or phenomena, assesses the code capability to scale them up, and evaluates the accuracy with which the code calculates them. The CSAU evaluation methodology consists of three primary elements.

The first element is requirements and code capabilities. In this element, scenario modeling requirements are identified and compared against code capabilities to determine the code's applicability to the particular scenario in a given plant design. In addition, an effort is made to identify potential code limitations. The modeling requirements are established by identifying and ranking processes and phenomena important to the particular scenarios (Phenomena Identification, Ranking Table, PIRT). The PIRT process provides a cost-effective approach to rank process and phenomena by evaluating their importance and modeling uncertainty relative to the primary safety criteria so that only the significant contributors need to be evaluated. [6] Code deficiencies and/or limitations are also identified and evaluated as to their potential effects on uncertainties to calculate primary safety criteria. The second element is assessment and ranging of parameters. In this element there are activities to assess the capability of the code to calculate processes important to the scenario by comparing calculations against experimental data to determine code accuracy, to determine scale-up capability, and to specify ranges of parameter variations needed for sensitivity studies. In addition, bounding analyses can be performed and, in such cases, code calculations may not be required. The third element is sensitivity and uncertainty analyses. The total uncertainty in a safety analysis includes contributors that arise from code limitations, scaling inaccuracies embedded in the experimental data (and therefore the code), and uncertainties associated with the state of the reactors at the initiation of a transient. This element contains the activities to calculate, collect, and combine individual contributors to uncertainty into the required total mean and $95 \%$ probability statements including separately identified and quantified biases.

Within the third element, different techniques for the uncertainty propagation can be used, including Monte Carlo analysis (MC), response surface (RS) methods, and statistical tolerance limits (TL). [5] Because of demanding calculation requirements, the MC method is currently not applicable to complex thermal-hydraulic codes. In the RS methods the RS replaces the code calculation in the MC analysis. The TL is approached using a random sampling of input parameters $\mathrm{N}$ times, and then using the computer code directly to generate $\mathrm{N}$ outputs that are used to estimate the actual uncertainty. Both RS and TL methods have been 
widely used to obtain the 95/95 (95\% probability with 95\% confidence level) PCTs and other design limit variables. The first CSAU demonstration by USNRC used the RS method while TL methods have gained popularity recently. The consideration of nonparametric tolerance limits was originally presented by Wilks [7]. Guba et. al. [8], Nutt and Wallis [9], and others studied and extended Wilks' method's applications in BEPU. Depending on conservativeness of selecting tolerance limit, the number of total random sample runs increases, with the most conservative method starts at 59. If more random runs are affordable, less conservative results can be obtained. For example, if a 93 run case is used, the second highest value is used to establish the 95/95 PCT instead of the highest value in the 59 run case.

\subsection{Limitations of Current CSAU Practices}

In spite of many successful applications, several aspects of CSAU have been criticized for the need of further improvement. [10] The critiques focus on two main issues - lack of objectiveness and high cost:

$\checkmark$ subjective judgement in PIRT process - PIRT process heavily relies on expert opinions.

$\checkmark$ high cost - due to heavily relying on large experimental database, needing many experts man-years work, and very high computational overhead. The first CSAU demonstration project spent about 13 man-years equivalent resource [1]. AREVA spent 40 staff-years to obtain NRC license for its BEPU large break LOCA analysis method [3].

$\checkmark$ mixing numerical errors with other uncertainties - due to limitations of two-phase models and numerics in the legacy system analysis codes currently used, it is impossible to separate numerical uncertainty from other uncertainties.

$\checkmark$ grid dependence and same numerical grids for both scaled experiments and real plants applications - since numerical errors cannot be quantified, one of ways to indirectly quantify them is to use same numerical grids for both scaled experiments and real plants applications. However, this is impossible or has large scaling distortion due to different scaling methods.

$\checkmark \quad$ user effects - different users can obtain different answers with same codes due to different discretization methods and different values for many users defined parameters.

$\checkmark$ Limits on uncertainty propagation - the current uncertainty quantification in CSAU is based on a "black box" approach with frozen codes. The simulation tool is treated as an unknown signal generator, a distribution of inputs is sent in and the distribution of the output is measured and correlated back to the original input distribution. Not all the important uncertainty in the codes can be accessed by users, i.e., heat transfer correlations in RELAP5. If the experts know the potential uncertainty but cannot explicitly perturb it through the code input, a bias is used and superposed on the results from statistical uncertainty analysis results. Using biases brings additional uncertainty on uncertainty qualification process.

Although large amount of efforts have been used to improve CSAU methodology as summarized by [5], the above issues still exist.

Many of those issues can be tracked down to the problems with the legacy safety analysis codes whose frameworks were developed long before CSAU was proposed. RELAP5 and TRAC were developed in 1970s. Even the new USNRC code TRACE is based on the frameworks of RELAP5 and TRAC. The fundamental two phase flow models in current codes are ill posed and only become well posed with the help of first order numerical diffusion errors [11]. The codes were initially designed to analyze the design basis accident LB-LOCA for the actively safe Generation II LWRs. After the Three Mile Island accident, time-consuming and costly modifications were performed to address SB-LOCA. Basing on 
large experimental database, tuning processes were widely used to produce an acceptable agreement between code calculations and a specific set of experimental data. The tuning procedures generate compensating errors, which limit the applicability of the codes to different reactor designs and accident scenarios. Due to these reasons, Zuber [12] believes that "the assessment of code modeling capabilities more a matter of faith than of reason."

\subsection{New Opportunities to Improve CSAU}

With the effort to develop next generation safety analysis codes such as the one being developed at INL [13], new opportunities appear to take advantage of new numerical methods, better physical models, and modern uncertainty qualification methods developed during the past three decades. Advances in numerical methods during the past three decades make it possible to perform simulations with at least second order accuracy in both time and space. New methods also enable implicitly coupled solution algorithms between coolant thermal hydraulics, solid conduction, and neutronics, which can greatly reduce numerical errors in simulations for very fast or very long transients $[14,15]$.

Contrary to the "black box" method, a more efficient uncertainty qualification approach can take advantage of intimate knowledge of the simulation code. In this "glass box" approach equations for the propagation of uncertainty are constructed and the sensitivities are solved for as variables in the simulation. This can generate similar sensitivity information as the above "black box" approach with fewer runs. There are two sensitivity analysis methods available: forward sensitivity method [16] and adjoint sensitivity method ([17], [18]). In the forward sensitivity method, the model is differentiated with respect to each parameter to yield an additional system of the same size as the original one, the result of which is the solution sensitivity. The gradient of any output variable depending on the solution can then be directly obtained from these sensitivities by applying the chain rule of differentiation. The forward sensitivity method is straight forward to derive and can handle both nonlinear and transient problems well. In the adjoint method, the solution sensitivities need not be computed explicitly. Instead, for each output variable of interest, one forms and solves an additional system, adjoint to the original one, the solution of which can then be used to evaluate the gradient of the output variable with respect to any set of model parameters. Adjoint method requires forming Jacobian matrix through complex mathematical derivation. It store solutions at each time step, so require huge storage for long transients. Adjoint methods have been successfully applied for linear steady state problems. However, huge challenge exists for transient non-linear problems. Some examples of using the adjoint method in system analysis applications can be found in the references ([17], [18], and [19]). The demonstrated examples shown in those references are simple test cases and it is not clear if adjoint analysis can be applied for design basis accidents such as LOCA.

This paper focuses on the forward sensitivity method. Forward sensitivity analysis (FSA) directly solves the PDEs (Partial Differential Equation) for parameter sensitivities defined as

$$
\mathbf{s}=\frac{d \mathbf{Y}}{d p}
$$

Here $\mathbf{Y}$ is the state vector and $p$ a constant physical parameter that the solution depends upon. Once we have solved for $\mathbf{Y}$ and $\mathbf{s}$ we can now estimate the solution $\mathbf{Y}$ uncertainty based on an uncertainty range in $p$ defined as $\Delta \mathrm{p}$,

$$
\mathbf{Y}_{ \pm} \approx \mathbf{Y} \pm \frac{d \mathbf{Y}}{d p} \Delta p=\mathbf{Y} \pm \mathbf{s} \Delta p
$$

The gradient information (s) can be taken advantage to avoid missing local maximum and save 
computational cost by reducing the total number of sampling as shown in Fig 1. In this figure, we are interested in finding the solution $Y$ sensitivity with a parameter $\mathrm{P}$. Assume that $\mathrm{P}_{\mathrm{M}}$ is the reference parameter value and $\left(\mathrm{Y}_{\mathrm{M}}, \mathrm{s}_{\mathrm{M}}\right)$ are the solutions for the physical problem and its sensitivity with $\mathrm{P}$. With the traditional black box method, one tends to take two bounding values for $\mathrm{P}$ to replace the many samplings in order to save computational cost. The first CSAU response surface was established in such way. For this arbitrary case, the black box method with 3 points samplings will naturally draw a wrong conclusion: $\mathrm{Y}$ is not sensitive to $\mathrm{P}$. However, with the sensitivity information available, one can easily estimates the local peak of $Y$ depending on $\mathrm{P}$ (marked by the star) and the local minimum value of $\mathrm{Y}$ depending on $\mathrm{P}$ according to Eq. (2).

The next section will describe the basic method of how to calculate the parameter sensitivities and how to extend the forward sensitivity method to include the time step and potentially the grid size as special sensitivity parameters so that the numerical errors can be quantified against uncertainties due to other physical parameters. This extension makes the forward sensitivity method a much more powerful tool to help uncertainty analysis in CSAU.

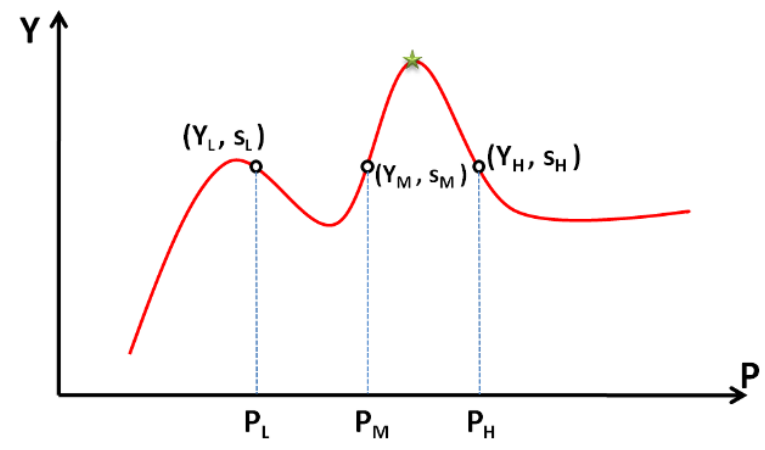

Fig 1 Schematic of How to Use Sensitivity Information.

\section{FORWARD SENSITIVITY ANALYSIS}

\subsection{Forward Sensitivity Method}

General forward sensitivity theory has been discussed in many references ([16], [20], [21], [22]). For completeness we summarize the basic ideas below. Reference [23] provides detailed description of FSA. Consider the general form of a nonlinear system of PDEs

$$
\frac{\partial \mathbf{Y}}{\partial t}-\mathbf{F}(t, \mathbf{Y}, p)=0
$$

Here $\mathrm{t}$ is time and $\mathbf{F}$ the vector of functions. By differentiating Eq. (3) with the parameter $p$, the equation for the time evolution of the sensitivity $\mathbf{s}$ can be obtained as:

$$
\frac{\partial \mathbf{s}}{\partial t}=\frac{\partial \mathbf{F}}{\partial \mathbf{Y}} \mathbf{s}+\frac{\partial \mathbf{F}}{\partial p}
$$

We will simplify notation to describe the solution procedure. Define residual functions:

$$
\begin{array}{r}
\mathbf{f}(\mathbf{Y})=\frac{\partial \mathbf{Y}}{\partial t}-\mathbf{F}(t, \mathbf{Y}, p) \\
\mathbf{g}(\mathbf{s})=\frac{\partial \mathbf{s}}{\partial t}-\frac{\partial \mathbf{F}}{\partial \mathbf{Y}} \mathbf{s}-\frac{\partial \mathbf{F}}{\partial p}
\end{array}
$$

We will solve the nonlinear system of equations

$$
\left[\begin{array}{c}
\mathbf{f}(\mathbf{Y}) \\
\mathbf{g}(\mathbf{s})
\end{array}\right]=0
$$


using Newton's method:

$$
\left[\begin{array}{ll}
\widetilde{\mathbf{J}} & \mathbf{0} \\
\mathbf{0} & \widetilde{\mathbf{J}}
\end{array}\right]\left[\begin{array}{c}
\boldsymbol{\delta} \mathbf{Y} \\
\boldsymbol{\delta} \mathbf{s}
\end{array}\right]=-\left[\begin{array}{c}
\mathbf{f}(\mathbf{Y}) \\
\mathbf{g}(\mathbf{s})
\end{array}\right]
$$

Eq. (8) will be iterated until the residuals of Eq. (7) are small. Here $\widetilde{\mathbf{J}}$ is the normal Jacobian matrix for the physical Eq. (5) and can be directly constructed:

$$
\widetilde{\mathbf{J}}=\frac{\partial \mathbf{f}}{\partial \mathbf{Y}}=\frac{\partial}{\partial \mathbf{Y}}\left(\frac{\partial \mathbf{Y}}{\partial t}\right)-\frac{\partial \mathbf{F}}{\partial \mathbf{Y}}
$$

or can be avoided with the Jacobian free method [16]:

$$
\begin{gathered}
\tilde{\mathbf{J}} \cdot \mathbf{v} \approx \frac{\mathbf{f}(\mathbf{Y}+\varepsilon \cdot \mathbf{v})-\mathbf{f}(\mathbf{Y})}{\varepsilon} \\
\frac{\partial \mathbf{F}}{\partial \mathbf{Y}} \mathbf{s}+\frac{\partial \mathbf{F}}{\partial p} \approx \frac{\mathbf{F}(t, \mathbf{Y}+\sigma \cdot \mathbf{s}, p+\sigma)-\mathbf{F}(t, \mathbf{Y}-\sigma \cdot \mathbf{s}, p-\sigma)}{2 \sigma}
\end{gathered}
$$

Where $\varepsilon$ and $\sigma$ are small perturbations and $\mathbf{v}$ a Krylov vector.

\subsection{Quantifying Numerical Errors with FSA}

There is an important assumption in using Eq. (2). This analysis requires that the numerical error in the solution method is small so that one is analyzing the physical model and not the numerical error. For this analysis the first step is to verify that the solution is not sensitive to the grid size $\Delta x$ or time step $\Delta t$. This can be done through two methods: the conventional time step and grid size convergence studies or taking $\Delta x$ and $\Delta t$ as special sensitivity parameters to calculate sensitivities of the solution with respect to them. The later method is more efficient than the conventional convergence study since they only require one run instead of the series runs required for a convergence study. We will describe this new method developed by us [23] to calculate $\Delta t$ sensitivity in this sub-section.

For the solution sensitivities with respect to the time step and the grid size, one can not directly derive the sensitivity equations from the original PDEs since the time and spatial steps are discrete quantities that depend on the discretization method. Let's consider the time step sensitivity. When we discretize a PDE, the actual discrete equation to be solved is the original PDE with added local truncation error (LTE). If we subtract the LTE term in the discretized PDE, the modified discretized equation will give us a higher order solution. The LTE is a function of the time step. Therefore, we can derive the time step sensitivity from the modified equation:

$$
\frac{\partial \mathbf{Y}}{\partial t}-\mathbf{F}(t, \mathbf{Y}, p)-\mathbf{L T E}(\Delta t)=0
$$

The forward sensitivity equation for the time step is,

$$
\mathbf{g}\left(\mathbf{s}_{\Delta t}\right)=\frac{\partial \mathbf{s}_{\Delta t}}{\partial t}-\frac{\partial \mathbf{F}}{\partial \mathbf{Y}} \mathbf{s}_{\Delta t}-\frac{\partial \mathbf{F}_{\mathrm{mod}}}{\partial(\Delta t)}
$$

The corresponding modified $\mathbf{F}$ function for the time step sensitivity problem should be:

$$
\mathbf{F}_{\text {mod }}(t, \mathbf{Y}, p, \Delta t)=\mathbf{F}(t, \mathbf{Y}, p)+\mathbf{L T E}(\Delta t)
$$

The second term in the right hind side of Eq. (13) can be approximated by the following equation:

$$
\frac{\partial \mathbf{F}}{\partial \mathbf{Y}} \mathbf{s}_{\Delta \mathbf{t}} \approx \frac{\mathbf{F}\left(x, t, \mathbf{Y}+\sigma \cdot \mathbf{s}_{\Delta \mathbf{t}}\right)-\mathbf{F}\left(x, t, \mathbf{Y}-\sigma \cdot \mathbf{s}_{\Delta \mathbf{t}}\right)}{2 \sigma}
$$

where $\mathbf{F}$ is the original function in Eq. (3) without adding the local time step truncation term; the third term in the right hind side of Eq. (13) can be analytically derived if the analytical form of LTE is available: 


$$
\frac{\partial \mathbf{F}_{\mathbf{m o d}}}{\partial \Delta t}=\frac{\partial \mathbf{L T E}(\Delta t)}{\partial \Delta t}
$$

Sometimes it is not easy to derive the analytical form of the LTE for complex time integration methods. In such case, we can use a numerical method to derive $\frac{\partial F_{\bmod }}{\partial(\Delta t)}$. The LTE has the following form:

$$
\operatorname{LTE}(\Delta t)=(\Delta t)^{q} \cdot \mathbf{f}_{\mathbf{L T E}}
$$

where $\mathrm{q}$ is the order of the time discretization scheme and $\mathbf{f}_{\mathbf{L T E}}$ a function not depending on $\Delta t$. The LTE can also be approximated by the difference of the current lower order scheme residual and a next higher order scheme residual:

$$
\operatorname{LTE}(\Delta t) \approx \operatorname{res}_{\mathrm{LO}}-\operatorname{res}_{\mathrm{HO}}
$$

where res $_{\mathbf{L O}}$ is the current lower order scheme residual and $\mathbf{r e s}_{\mathbf{H O}}$ a higher order scheme residual. According to Eqs. (17) and (18), we can derive

$$
\frac{\partial \mathbf{F}_{\text {mod }}}{\partial(\Delta t)} \approx-q \frac{\text { res }_{\mathbf{L O}}-\text { res }_{\mathbf{H O}}}{\Delta t}
$$

Within each time step iteration we first converge the physical solution and then we calculate the parameter sensitivities. Therefore by the time we use Eq. (19), the lower order scheme residual is already converged and is very close to zero. So we can further simplify Eq. (19) to

$$
\frac{\partial \mathbf{F}_{\text {mod }}}{\partial(\Delta t)} \approx q \frac{\text { res }_{\mathbf{H O}}}{\Delta t}
$$

Eq. (20) defines a general method which is suitable for any time step schemes. We call this method the residual difference method. The time step sensitivity here reflects the accumulated time step error instead of LTEs. The accumulated time step error (global error) usually cannot be obtained except for special cases with analytical solutions. The spatial step sensitivity equation can be derived with a similar method.

\subsection{FSA Example}

\subsubsection{Thermal wave problem}

We will use the thermal wave problem [24] as a simple example to show the extended forward sensitivity method. This problem has very useful analytical solutions for the problem itself and all the parameter sensitivities so that the numerical algorithms can be accurately verified. The following is the equation for the thermal wave problem:

$$
\frac{\partial T}{\partial t}-\frac{\partial^{2} T}{\partial x^{2}}=f(T, c, \delta)
$$

for $x \in(-\infty,+\infty)$, and $t \geq 0$. We assume an exact solution which describes a thermal wave moving with a constant velocity $\mathrm{c}$ and with a constant wave width $\delta$ :

$$
T(x, t, c, \delta)=\frac{1}{2}\left(1-\tanh \left(\frac{x-c t}{\delta}\right)\right)
$$

In Eq. (21), $f(T, c, \delta)$ is the source term for the manufactured solution, which can be calculated by substituting the assumed solution T (Eq. (22)) into the left hind side of Eq. (21):

$$
f(T, c, \delta)=\frac{2 T(1-T)(c \delta-2+4 T)}{\delta^{2}}
$$

The reference values for $\mathrm{c}$ and $\delta$ are: $\mathrm{c}=2, \delta=1$. In the numerical analysis, we will limit the computation domain to $[-10,10]$. The boundary conditions and initial condition will be directly 
derived from the analytical solution. So are for the solution sensitivity with respect to parameter $\mathrm{c}$, and the solution sensitivity with respect to the parameter $\delta$.

\subsubsection{Time step sensitivity analytical solution}

Now we will discuss the modified equation for the first order backward Euler scheme (BE) and the associated analytical solution for the time step sensitivity. The local truncation error is

$$
L T E_{t}=\frac{\Delta t}{2} \frac{\partial^{2} T}{\partial t^{2}}
$$

and the modified equation is

$$
\frac{\partial T}{\partial t}-\frac{\partial^{2} T}{\partial x^{2}}-\frac{\Delta t}{2} \frac{\partial^{2} T}{\partial t^{2}}=f(T)
$$

The analytical solution for Eq. (25) with the reference values of $\mathrm{c}=2$ and $\delta=1$ can be derived [25]:

$$
T_{M E A}(x, t, \Delta t)=\frac{1}{2}\left(1-\tanh \left(\frac{x-\frac{2}{\sqrt{1-2 \Delta t}} t}{\frac{1}{\sqrt{1-2 \Delta t}}}\right)\right)
$$

Comparing this equation with Eq. (22), one can see that this solution represents a thermal wave with the width $1 / \sqrt{1-2 \Delta t}$ and speed $2 / \sqrt{1-2 \Delta t}$. The first order temporal error is equivalent to simultaneously perturbing "c" and " $\delta$ " by dividing $\sqrt{1-2 \Delta t}$. According to Eq. (26), we can derive an analytical solution for time step sensitivity for the $1^{\text {st }}$ order backward Euler scheme:

$$
s_{\Delta t}(x, t, \Delta t)=\frac{x\left(1-(\tanh (x \sqrt{1-2 \Delta t}-2 t))^{2}\right)}{2 \sqrt{1-2 \Delta t}}
$$

Eq. (27) will be used as the analytical solution to check the accuracy of the numerical method.

\subsubsection{Sensitivity comparison}

Both time and spatial steps convergence studies for the physical problem, parameter sensitivities, and time step sensitivity were performed. The numerical results verify correct convergence speeds for all the problems.

The extended forward sensitivity analysis provides a systematic method to evaluate the parameter sensitivity effects on solution, along with time and space convergence information. Fig. 2 compares sensitivity effects from time step, the thermal wave speed, and the thermal wave width parameters for a large time step $(0.1)$ and a very small spatial step $(1 / 32)$. We can readily notice that the solution is still very sensitive to the time step, which means the solution is not well converged. The uncertainty bar from the time step is much larger than the uncertainty bar from the thermal wave width parameter and is at the same order with the uncertainty bar from the thermal wave speed parameter. Therefore, the solution along with the sensitivity analysis is not reliable. One should use a smaller time step in order to obtain accurate solution and sensitivity analysis results. Fig. 3 shows the similar results for a very small time step $(0.001)$ and the same small spatial step (1/32). In this case, the solution is not sensitive to the time step anymore; therefore the solution and sensitivity results are reliable. These comparisons show how important it is to consider numerical errors when performing uncertainty and sensitivity analysis. In conventional Monte Carlo type of uncertainty analysis, one tends to use large time steps and coarse grids in order to perform large amount of calculations, without considering numerical errors. The sensitivity results from such practices often contain large 
error and even the trends are wrong. Including the time step and the grid size as special sensitivity parameters provides a new method to avoid such pitfalls and improves both accuracy and efficiency.

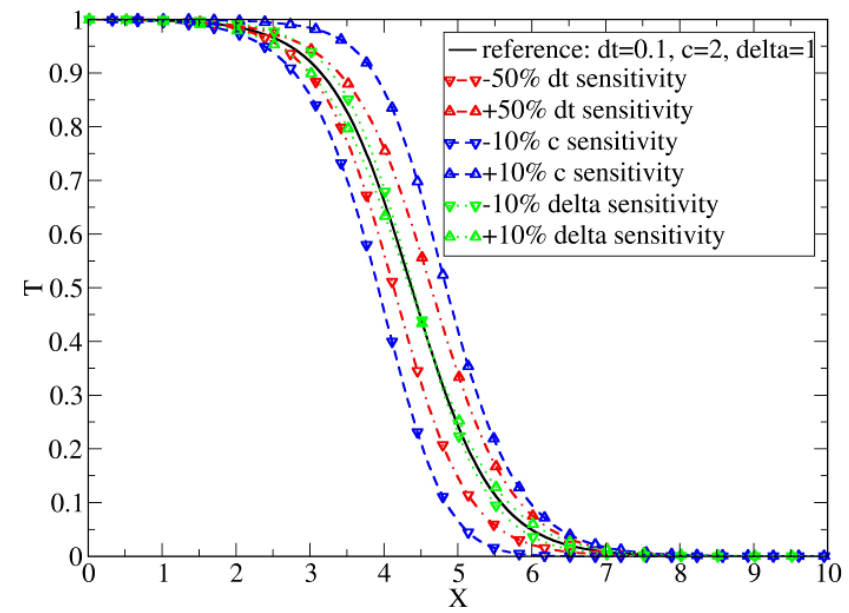

Fig 2 Comparison of Sensitivity Effects from Time Step, Wave Speed, and Wave Width for the Thermal Wave Problem, T=2, Backward Euler Scheme, $\Delta x=1 / 32, \Delta t=0.1$.

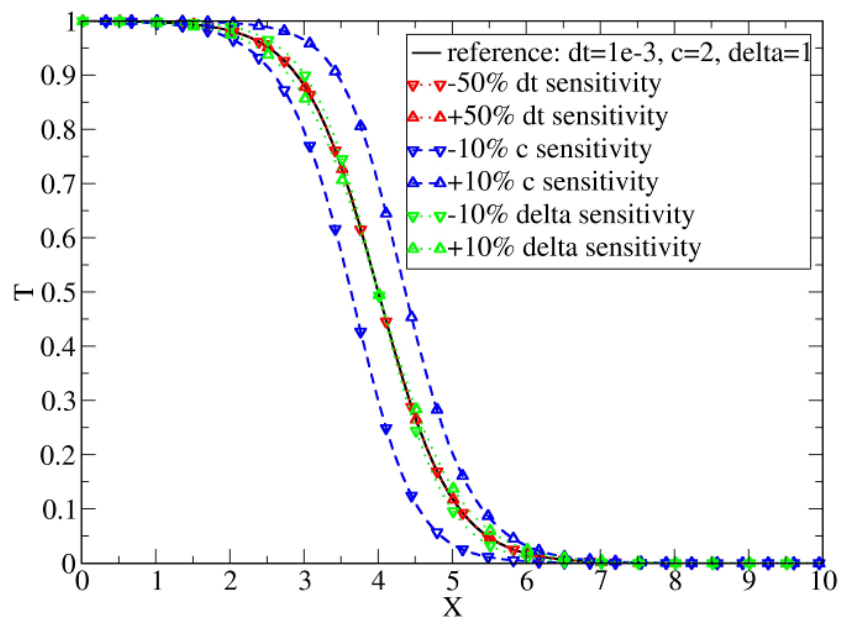

Fig 3 Comparison of Sensitivity Effects from Time Step, Wave Speed, and Wave Width for the Thermal Wave Problem, T=2, Backward Euler Scheme, $\Delta x=1 / 32, \Delta t=0.001$.

\section{APPLYING FSA IN CSAU}

\subsection{Potential Improvements on CSAU with FSA}

The FSA method discussed in the previous section can be implemented in a new advanced system analysis code, [13], so that CSAU could be significantly improved by reducing its cost and subjective judgement. Some of potential improvements include:

$\checkmark$ quantifying numerical errors and reducing computational costs by choosing optimized time steps and spatial sizes; 
$\checkmark$ reducing computational cost by using gradient information (sensitivity result) to reduce sampling number;

$\checkmark$ enabling a quantitative PIRT (Q-PIRT) to reduce subjective judgement, reduce experts cost, and improve efficiency;

$\checkmark \quad$ allowing grid independence for scaled integral effect test (IET) simulation and real plant applications by eliminating numerical uncertainty on scaling;

$\checkmark$ greatly reduce user effects by not letting users select the grid size and time step.

Existing reactor safety analysis codes cannot quantify numerical errors. The numerical errors are mixed with all other error sources and uncertainties together, and the total simulation uncertainties can only be partially revealed by IET experimental data. While typical CFD calculation can quantify numerical errors by performing time and space steps convergence studies, numerical errors are often made extremely small. It just wastes computational resource if numerical errors are many orders smaller than physical model uncertainties. Instead, time and space discretizations should be chosen so that numerical errors are reasonably smaller than important physical parameter uncertainties. As described in previous section, the FSA approach can be adapted to address global spatial and temporal truncation errors. This allows one to place numerical uncertainties and physical parameter uncertainties on the same level so that they can be directly compared. The concerned physical model uncertainties therefore naturally set allowable numerical errors: the time step and grid size is not too large to distort the real physics model uncertainty analysis result and is not too small to require unnecessary over-computation.

Experienced experts opinions are highly valuable to guide safety analysis, therefore are indispensable in CSAU. However, the subjective judgement should be verified by less expensive and objective methods. Additionally, the expense of assembling a group of top experts for extended time becomes increasingly higher. On the other hand, the computational cost is decreasing at exponential speed. A Q-PIRT process can complement the traditional experts-based PIRT process with objective results and less experts' time replaced by inexpensive computer run time. With the FSA method, a Q-PIRT process can be implemented with following steps:

$\checkmark$ Pre-run: choose proper large time and space steps to perform a quick run. The time and step steps should fall in the asymptotic region and be smaller than the physical dynamical scales.

$\checkmark$ Parameter ranking and time and space steps selection:

○ Rank parameters according to their uncertainty importance:

$$
\left|s_{p_{1}} \cdot \Delta p_{1}\right| \geq\left|s_{p_{2}} \cdot \Delta p_{2}\right| \geq\left|s_{p_{3}} \cdot \Delta p_{3}\right| \geq \cdots
$$

○ Only keep parameters with important uncertainty effects in subsequent sensitivity analysis $\left(p_{1}, p_{2}, \cdots, p_{\text {last }}\right)$;

- Choose proper time and space steps so that the numerical errors are much smaller than the minimal uncertainty caused by the physical parameters in the list, i.e.,

$$
\left|s_{\Delta t} \cdot\left(\Delta t-\Delta t_{\text {small }}\right)\right| \leq 1 \% \cdot\left|s_{p_{\text {last }}} \cdot \Delta p_{\text {last }}\right|, \quad\left|s_{\Delta x} \cdot\left(\Delta x-\Delta x_{\text {small }}\right)\right| \leq 1 \% \cdot\left|s_{p_{\text {last }}} \cdot \Delta p_{\text {last }}\right|
$$

$\checkmark$ Detailed run: generate accurate solution and sensitivity information for the selected parameters according to the proper time and space steps.

\subsection{Requirements on the New Code}

With all the potentials discussed in the above subsection, there are also requirements on the new code to fully take advantage of FSA methods. The following discussions highlight some important requirements to implement FSA. 
$\checkmark$ All physical models must be smooth; otherwise, we end up with infinite large sensitivity at the non-smooth point. Non-smooth models often appear in closure models and control systems in traditional system analysis codes. Those non-smooth models are one of major reasons limiting FSA application in system analysis codes.

$\checkmark$ For those component models containing large amount of parameters, one or few of lumped parameters should be provided so that layered sensitivity analysis can be performed to limit the total number of parameters in a reasonable range. As an example, the new INL code will be designed to have a multi-fidelity modeling structure. Lower fidelity models with few of parameters will be tried first; if any lower fidelity models have been identified to have large uncertainty, higher fidelity models will be used to reduce simulation uncertainty.

$\checkmark$ Physical parameter uncertainty distribution information should be included in the code so that internal uncertainty qualification can be implemented in the code instead of requiring users to provide such information. For current system analysis codes, part of that information are often included in the code manual, but not written into a code. Current uncertainty qualification applications use those uncertainty information backward if the codes allow modifying those parameters.

There are also other important requirements, such as advanced numerical techniques, efficient code structure designs, well-posed PDEs, etc. Due to page limits, no further discussion is provided.

\section{CONCLUSIONS}

Code Scaling, Applicability, and Uncertainty (CSAU) methodology could be significantly improved with forward sensitivity analysis implemented in a new advanced system analysis code by reducing its cost and subjective judgement. By including time step and grid size sensitivity analysis, time and space convergence studies can be performed in one run and global numerical errors can be directly compared with physical parameter uncertainties. A quantitative PIRT process can be implemented with forward sensitivity analysis. Effectively using forward sensitivity raises new requirements on system analysis code design.

\section{ACKNOWLEDGMENTS}

This work was supported through INL Laboratory Directed Research and Development program under DOE Idaho Operations Office Contract DE-AC07-05ID14517.

\section{REFERENCES}

1. B. E. Boyack, et. al., "Quantifying Reactor Safety Margins," papers 1 to 6, Nuclear Engineering and Design, Vol. 119, (1990).

2. M.Y. Young, et. al., "Application of Code Scaling Applicability and Uncertainty Methodology to the Large Break Loss of Coolant," Nuclear Engineering and Design, Vol. 186, pp. 39-52, (1998).

3. R.P. Martin and L.D. O'Dell, “AREVA's Realistic Large Break LOCA Analysis Methodology," Nuclear Engineering and Design, Vol. 235, pp. 1713-1725, (2005).

4. K. Muftuoglu, et. al., "Comparison of Realistic Large Break LOCA Analyses of a 3-Loop Westinghouse Plant Using Response Surface and Statistical Sampling Techniques," Proc. 12th Int. Conf. Nuclear Engineering (ICONE-12), Arlington, Virginia, ASME, (2004).

5. A. Prosek and B. Mavko, "The State-of-the-Art Theory and Applications of Best-Estimate Plus Uncertainty Methods," Nuclear Technology, Vol. 158, pp. 69-79, (2007).

6. G.E. Wilson and B.E. Boyack, "The Role of the PIRT Process in Experiments, Code 
Development and Code Applications Associated with Reactor Safety Analysis," Nuclear Engineering and Design, Vol. 186, pp. 23-37, (1998).

7. S. S. Wilks, "Determination of Sample Sizes for Setting Tolerance Limits," Ann. Math. Stat., Vol. 12, pp. 91 (1941).

8. A. Guba, et. al., "Statistical aspects of best estimate method - I," Reliability Engineering \& System Safety, Vol. 80, pp. 217-232, (2003).

9. W.T. Nutt, G.B. Wallis, "Evaluation of nuclear safety from the outputs of computer codes in the presence of uncertainties," Reliability Engineering \& System Safety, Vol. 83, pp. 57-77, (2004).

10. G.E. Wilson, et. al., "TPG Response to the Foregoing Letters-to-the-Editor," Nuclear Engineering and Design, Vol. 132, pp 431-436, (1992).

11. N.T. Dinh, et. al., "Understanding the Ill-posed Two-fluid Model," Proceedings of the 10th International Topical Meeting on Nuclear Reactor Thermal Hydraulics (NURETH10), Seoul, Korea, October, (2003).

12. N. Zuber, "The Effects of Complexity, of Simplicity and of Scaling in Thermal-Hydraulics," Nuclear Engineering and Design, Vol. 204, n 1-3, pp 1-27, (2001).

13. N.T. Dinh, et. al., "A Next Generation of Nuclear Plant System Analysis Codes to Support Risk-Informed Safety Margin Characterization," Proceedings of the 13th International Topical Meeting on Nuclear Reactor Thermal Hydraulics (NURETH13), Kanazawa, Ishikawa-Ken, Japan, (2009).

14. V.A. Mousseau, "Accurate Solution of the Nonlinear PDE's from Thermal Hydraulics," Nuclear Technology. Vol. 158, pp. 26-35, (2007).

15. D.A. Knoll and D.E. Keyes, "Jacobian-Free Newton-Krylov Methods: A Survey of Approaches and Applications," Journal of Computational Physics, Vol. 193, pp. 357-397, (2004).

16. A.C. Hindmarsh, et. al., "SUNDIALS: Suite of Nonlinear and Differential/Algebraic Equation Solvers," ACM Transactions on Mathematical Software, Vol. 31, No. 3, pp. 363-396, (2005).

17. D.G. Cacuci, and M. Ionescu-Bujor, "Adjoint Sensitivity Analysis of the RELAP5/MOD3.2 Two-Fluid Thermal-Hydraulic Code System-I: Theory," Nuclear Science and Engineering, Vol. 136, pp. 59-84, (2000).

18. M. Ionescu-Bujor, and D.G. Cacuci, "Adjoint Sensitivity Analysis of the RELAP5/MOD3.2 Two-Fluid Thermal-Hydraulic Code System-II: Applications," Nuclear Science and Engineering, Vol. 136, pp. 85-121, (2000).

19. C. Perret, and F. Barré, "Validation of the DASM Method Used for Sensitivity Analysis and Uncertainty Evaluation in CATHARE 2," Proceedings of NURETH 8. Kyoto, Japan, (1997).

20. M.D. Tocci, "Sensitivity Analysis of Large-Scale Time Dependent PDEs," Applied Numerical Mathematics, Vol. 37, pp. 109-125 (2001).

21. S.L. Lee, et. al., "Analyzing Radiation Diffusion Using Time-Dependent Sensitivity-Based Techniques," Journal of Computational Physics, Vol. 192, pp. 211-230 (2003).

22. K.J. Dowding, B.F. Blackwell, "Sensitivity Analysis for Nonlinear Heat Conduction," Journal of Heat Transfer, Vol. 123, pp. 1-10 (2001).

23. H. Zhao and V.A. Mousseau, Extended Forward Sensitivity Analysis for Uncertainty Quantification, INL technical report, INL/EXT-08-14847, September (2008).

24. O. Knio, et. al., "A Semi-implicit Numerical Scheme for Reacting Flow: II. Stiff Operator-split Formulation," Journal of Computational Physics, Vol. 154, pp. 428-467 (1999).

25. Rick Rauenzahn, Los Alamos National Laboratory, personal communication (2008). 